\title{
Transplantation of angiogenin-overexpressing mesenchymal stem cells synergistically augments cardiac function in a porcine model of chronic ischemia
}

\author{
Sheng-Dong Huang, MD, PhD, ${ }^{a}$ Fang-Lin Lu, MD, PhD, ${ }^{a}$ Xun-Yu Xu, MD, PhD, ${ }^{a}$ Xiao-Hong Liu, MD, PhD, ${ }^{a}$ \\ Xian-Xian Zhao, MD, PhD, ${ }^{\text {b }}$ Bao-Zhen Zhao, MD, PhD, ${ }^{\mathrm{c}}$ Li Wang, MD, PhD, ${ }^{\mathrm{d}}$ De-Jun Gong, MD, PhD, ${ }^{\mathrm{a}}$ \\ Yang Yuan, MD, PhD, and Zhi-Yun Xu, MD, PhD ${ }^{a}$
}
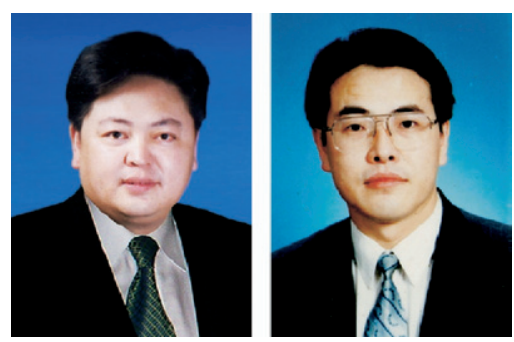

Drs Huang and Xu (left to right).
From the Institute of Thoracic Cardiac Surgery ${ }^{\mathrm{a}}$ and the Departments of Cardiology, ${ }^{\mathrm{b}}$ Ultrasound, ${ }^{\mathrm{c}}$ and Radiology, ${ }^{\mathrm{d}}$ Changhai Hospital Second Military Medical University, Shanghai, People's Republic of China.

Supported by the National Natural Science Foundation of China (grant 30371418 and 30471718).

Received for publication May 12, 2006; revisions received July 7, 2006

Address for reprints: Zhi-Yun Xu, MD, $\mathrm{PhD}$, Institute of Thoracic Cardiac Surgery, Changhai Hospital, 174 Changhai Rd, Shanghai, 200433, People's Republic of China (E-mail: zhiyunxu@smmu.edu.cn).

J Thorac Cardiovasc Surg 2006;132:1329-38 $0022-5223 / \$ 32.00$

Copyright $\odot 2006$ by The American Association for Thoracic Surgery

doi:10.1016/j.jtcvs.2006.08.021
Objective: Accumulated evidence suggests that myogenesis and angiogenesis induced by implanted cells play important roles in restoring cardiac function after a myocardial infarction. The current study investigated the effects of transplanted autologous mesenchymal stem cells overexpressing angiogenin on myocardial perfusion and cardiac function in the porcine chronic ischemic model.

Methods: Chronic ischemia was generated in Yorkshire pigs by placing an ameroid constrictor around the left circumflex artery. Four weeks after occlusion, the animals were randomly separated into 4 groups: pigs in the MSC $^{\text {AdAng }}$ or MSC ${ }^{\text {AdNull }}$ groups were implanted with $6 \times 10^{8}$ mesenchymal stem cells infected with adenovirus containing angiogenin gene or null adenovirus, respectively; pigs in the AdAng or AdNull groups were injected intramyocardially with adenovirus $\left(5 \times 10^{9}\right.$ plaque forming unit/pig) containing angiogenin gene or null adenovirus, respectively. Four weeks after implantation, mesenchymal stem cells prelabeled with DiI were observed within the implanted area in both cell transplantation groups.

Results: Angiogenin protein levels were significantly greater in the $\mathrm{MSC}^{\text {AdAng }}$ and AdAng groups than in the other 2 groups and were associated with greater neovessel formation than in the other 2 groups. Mesenchymal stem cell transplantation decreased scar size and increased scar thickness. Both the AdAng and MSC ${ }^{\text {AdNull }}$ groups experienced improved cardiac function compared with that seen in the AdNull group. However, a synergistic effect of mesenchymal stem cells and angiogenin was observed in the MSC ${ }^{\text {AdAng }}$ group because myocardial perfusion and cardiac function increased significantly $(P<.05$ for all groups) in this group compared with all the others.

Conclusions: Transplantation of autologous mesenchymal stem cells transfected with the angiogenin gene revealed a synergistic effect on the improvement of heart perfusion and function after ameroid occlusion.

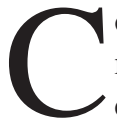
oronary occlusions can result in congestive heart failure if the heart cannot mount an adequate angiogenic response to restore perfusion to the ischemic cardiomyocytes. The implantation of cells into the ischemic myocardium might restore function by inducing angiogenesis and preventing wall thinning and scar expansion..$^{1-5}$ Augmentation of cell transplantation with proteins or genes might enhance the regeneration achieved with this therapy. However, few studies have reported a synergistic effect of cell and gene therapy on heart function in an ischemic cardiomyopathic model.

Mesenchymal stem cells (MSCs) possess pluripotential capabilities ${ }^{6}$ and are negative for hematopoietic lineage cell markers, including CD34 and CD45. ${ }^{6,7}$ MSCs are easily obtained from bone marrow and can be expanded in culture and 


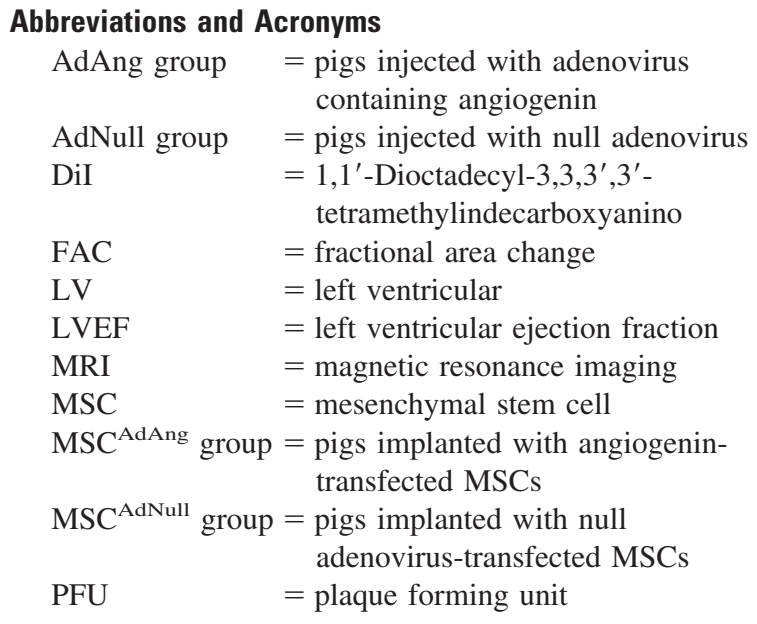

then cryopreserved for future use. ${ }^{8-11}$ Encouraging preclinical data have established the feasibility of autologous marrow cell transplantation, and several clinical studies have been initiated ${ }^{12,13}$ suggesting that MSCs might be the ideal donor cells for cellular cardiomyoplasty.

Angiogenin is a basic heparin-binding protein of 14.4 $\mathrm{kd}$ that stimulates angiogenesis. ${ }^{14} \mathrm{We}$ have successfully spliced the human angiogenin gene into an adenovirus that can increase vessel density when implanted into ischemic hearts. ${ }^{15,16}$ Transfection by means of direct injection of the gene into the myocardial tissue is low, but transplanted cells can alternatively be used as carriers for gene transfer. In this case cells were transfected in vitro with the angiogenin gene to enhance gene expression after implantation into damaged tissue.

In the present study we directly compared the effects of autologous MSC transplantation therapy and angiogenin gene therapy, alone and in combination, on the restoration of cardiac function in the ischemic heart in an adult pig model. By using clinically relevant evaluation techniques, we showed that both cell therapy and gene therapy improved cardiac function. However, the combination treatment synergistically enhanced cardiac function. Our data provide important information concerning the potential clinical application of genetically modified cell transplantation to treat patients with ischemic heart disease.

\section{Materials and Methods Experimental Animals}

Yorkshire pigs (20-25 kg) were obtained from Shanghai Experimental Animal Supply, China. The animal protocol was approved by the Institutional Animal Care and Utilization Committee of the Second Military Medical University. All operative procedures were performed according to the "Canadian Council on Animal Care and Use of Laboratory Animals," published by the National Institute of Health.

\section{Animal Model}

A porcine model of chronic myocardial ischemia was induced by using ameroid constrictors. Forty pigs were sedated with ketamine hydrochloride (15 mg/kg administered intramuscularly) and anesthetized with pentobarbital sodium $(10 \mathrm{mg} / \mathrm{kg}$ administered intravenously). The animals were then intubated and ventilated with oxygen containing isoflurane ( $0.8 \%$ to $1.5 \%)$. In each animal the iliac crest area was prepared by using sterile techniques, and approximately $14 \mathrm{~mL}$ of bone marrow was aspirated into a syringe containing 6000 units of heparin for MSC isolation and culture.

A left thoracotomy was then performed, and an ameroid constrictor $(2.25-2.50 \mathrm{~mm}$ diameter matched to the diameter of the artery; Research Instruments SW) was placed around the proximal left circumflex branch of the left coronary artery. The pericardium and the chest were closed. The animals were treated postoperatively with amoxicillin (500 $\mathrm{mg}$ administered orally twice daily) and buprenorphine $(0.3 \mathrm{mg}$ administered intramuscularly twice daily) for 3 days.

\section{Isolation and Expansion of MSCs}

The bone marrow underwent gradient centrifugation. Mononuclear cells were collected and cultured in cell culture media (DM-10) supplemented with $1 \%$ penicillin-streptomycin and $10 \%$ fetal bovine serum. The cells were incubated with antibodies against CD34 and CD45, and these cells were removed by passing through a magnetic column (Miltenyi Biotech). The CD $34^{-} / \mathrm{CD}^{-} 5^{-} \mathrm{MSCs}$ were collected, ${ }^{7}$ cultured, and subcultured for transplantation.

\section{Angiogenin Gene Transfection and Cell Labeling}

Replication-deficient recombinant adenovirus containing the human angiogenin gene was constructed. One day before transplantation, the MSCs were infected with the adenovirus at 50 plaque forming unit (PFU)/cell. Null adenovirus was used to infect MSCs as a control group. Some of the cultured MSCs were infected with adenovirus containing green fluorescence protein gene to examine the transduction efficiency. On the day of implantation, the MSCs were also labeled with 1,1' -Dioctadecyl-3,3,3',3'-tetramethylindecarboxyanino (DiI), according to the manufacturer's protocol. The labeled cells were washed, detached from the culture dish, centrifuged, and suspended in culture media containing 1000 units of heparin $\left(6 \times 10^{8}\right.$ cells $\left./ \mathrm{mL}\right)$ for transplantation.

\section{MSC or Gene Implantation}

Four weeks after ameroid constrictor implantation, occlusion of the target artery was confirmed by means of angiography. Forty pigs were randomly assigned to one of 4 experimental groups, as follows: group 1, 10 pigs implanted with angiogenin-transfected MSCs (MSC ${ }^{\text {AdAng }}$ group; $6.0 \times 10^{8}$ cells per pig); group 2, 10 pigs implanted with null adenovirus-transfected MSCs (MSC ${ }^{\text {AdNull }}$ group; $6.0 \times 10^{8}$ cells per pig); group 3, 10 pigs injected with adenovirus containing angiogenin (AdAng group, $5 \times 10^{9} \mathrm{PFU} /$ pig); and group 4, 10 pigs injected with null adenovirus (AdNull group, $5 \times 10^{9} \mathrm{PFU} / \mathrm{pig}$ ). The cells or viral solutions were intramyocardially injected with a 30-gauge needle at 9 locations within the ischemic myocardial area through an open chest incision. After implantation, the chest was closed, and each animal was recovered as previously described. 
Coronary Angiographic Analysis of Collateral Density Coronary angiography was performed before and 4 weeks after cell or virus implantation to confirm occlusion and formation of collateral vessels. After achievement of general anesthesia, a $7 \mathrm{~F}$ catheter (Britetip JL4 or Cordis) was inserted into the right coronary artery. Contrast medium $(4.5 \mathrm{~mL})$ was injected at a rate of 1.5 $\mathrm{mL} / \mathrm{s}$, and digitally subtracted images were obtained. These same procedures were repeated for the left coronary artery. Evaluation of angiographic collateral density was performed through cine film reviewed by 2 experienced angiographers who were blinded to the experimental groups by using a Rentrop score from 0 to $3(0=$ no visible collateral vessels; $1=$ faint filling of side branches of the main epicardial vessel without filling the main vessel; $2=$ partial filling of the main epicardial vessel; $3=$ complete filling of the main vessel). ${ }^{17}$

\section{Echocardiographic Analysis of Regional and Global Heart Function}

Echocardiographic images (Hewlett Packard) were obtained in the open-chest state before and 4 weeks after treatment. Images were compared by using apical 4-chamber and midventricular short-axis planes. Left ventricular (LV) end-diastolic volume (EDV) and LV end-systolic volume (ESV) were measured, and LV ejection fraction (LVEF) was calculated by using the following equation: $L V E F=(E D V-E S V) / E D V \times 100 \%$. Ventricular function was also evaluated as fractional area change (FAC) of the left ventricle. FAC was measured by tracing the end-diastolic area (EDA) and the end-systolic area (ESA) of the ventricle from the midpapillary short-axis view and then was calculated as follows: $F A C=$ $([E D A-E S A] / E D A) \times 100 \%$. Regional wall thickening was determined by measuring lateral-posterior wall thickness at end diastole and end systole and using the following equation:

Wall thickening $=($ Systolic thickness - Diastolic thickness $) /$

Systolic thickness $\times 100 \%$.

\section{Magnetic Resonance Imaging}

Magnetic resonance imaging (MRI) was performed before and 4 weeks after treatment. Each animal was sedated with ketamine hydrochloride ( $5 \mathrm{mg} / \mathrm{kg}$ administered intramuscularly) and diazepam (10 mg per pig administered intramuscularly) and placed in a prone position with a 4-channel, quadrature body, phased-array coil positioned over the back. Basal, midventricular, and apical images were collected simultaneously. For perfusion analysis, the LV image was divided into 16 segments according to the American Heart Association model. ${ }^{18}$ Three representative short-axis slices obtained at the level of the apex, midventricle, and base were divided into 4, 6, and 8 segments, respectively. The perfusion was analyzed in each segment.

\section{Heart Morphologic Study and Myocardial Histology}

At the end of the study, the hearts were excised and sliced into 5 or 6 rings. Both sides of the slices were photographed. The infarct area and normal region of each slice were determined by using the Scion Image Analysis program. The percentage of infarction of the whole LV wall was calculated.

The myocardial biopsy specimens were collected and frozen immediately. The tissues were serially sectioned at $5 \mu \mathrm{m}$. The samples were stained with hematoxylin and eosin and for Factor VIII to identify endothelial cells in the blood vessels. Both endothelial cells and vascular structure were evaluated by using fluorescent and confocal microscopy (PCM 2000, Nikon).

\section{Secretion of Angiogenin in Gene-modified MSCs}

A portion of angiogenin-modified MSCs were collected from the implanted cell suspension. The cells were cultured for 8 days, and the cultured media were collected for quantification of angiogenin protein levels by using enzyme-linked immunosorbent assay, according to the manufacturer's protocol. Media cultured with nontransfected MSCs were used as controls.

\section{Western Blotting Analysis}

At the end of the study, ventricular muscle was harvested adjacent to the injection site for the evaluation of angiogenin protein levels. The samples were homogenized in a lysis buffer and centrifuged at $12,000 \mathrm{~g}$ for 10 minutes at $4^{\circ} \mathrm{C}$. The supernatant was collected, and protein concentrations were measured. Total protein (40 $\mu \mathrm{g} / \mathrm{lane})$ was used for Western blot analysis with rabbit anti-angiogenin polyclonal IgG. Densitometry measurements were performed on digitized images of the immunoprobed membranes by using ImageQuant software (Molecular Dynamics).

\section{Statistical Analyses}

All results were presented as means \pm standard deviation. Continuous variables (LVEF, FAC, regional wall thickening, LV infarction area, angiogenin expression, and blood vessel density) were compared with unpaired Student $t$ tests (for comparison between 2 groups) or analysis of variance for comparison between multiple groups. Comparison of angiographic collateral density through the collateral index was assessed by using 2-sided Kruskal-Wallis (multiple group comparison) and Wilcoxon rank sum (2 group comparison) tests.

\section{Results}

\section{In Vitro Studies}

MSCs $\left(\mathrm{CD} 34^{-} / \mathrm{CD} 45^{-}\right)$were cultured for transplantation. Flow cytometric analysis showed that most cultured MSCs expressed CD44 (96.2\% $\pm 1.2 \%)$. An adenoviral system was used to deliver angiogenin into the MSCs with a transfection efficiency of approximately $100 \%$. MSCs from the MSC $^{\text {AdAng }}$ and MSC ${ }^{\text {AdNull }}$ groups were cultured for 8 days to quantify the secretion of angiogenin from the transfected cells. The angiogenin protein level in the media cultured with MSC $^{\text {AdAng }}(1245 \pm 153 \mathrm{pg} / \mathrm{mL})$ was significantly greater $(P=.001)$ than in the control media cultured with $\operatorname{MSC}^{\text {AdNull }}(30 \pm 3 \mathrm{pg} / \mathrm{mL})$.

\section{In Vivo Studies}

Eight pigs died of an infection (MSC ${ }^{\text {AdAng }}$ group, $\mathrm{n}=1$; MSC $^{\text {AdNull }}$ group, $\mathrm{n}=2$; AdAng group, $\mathrm{n}=3$; AdNull group, $n=2$ ) and were therefore removed from the study.

Coronary angiography was performed at 28 days after ameroid implantation (Figure 1,Al-A4). There was no difference in angiographic (Rentrop) scores among the 4 

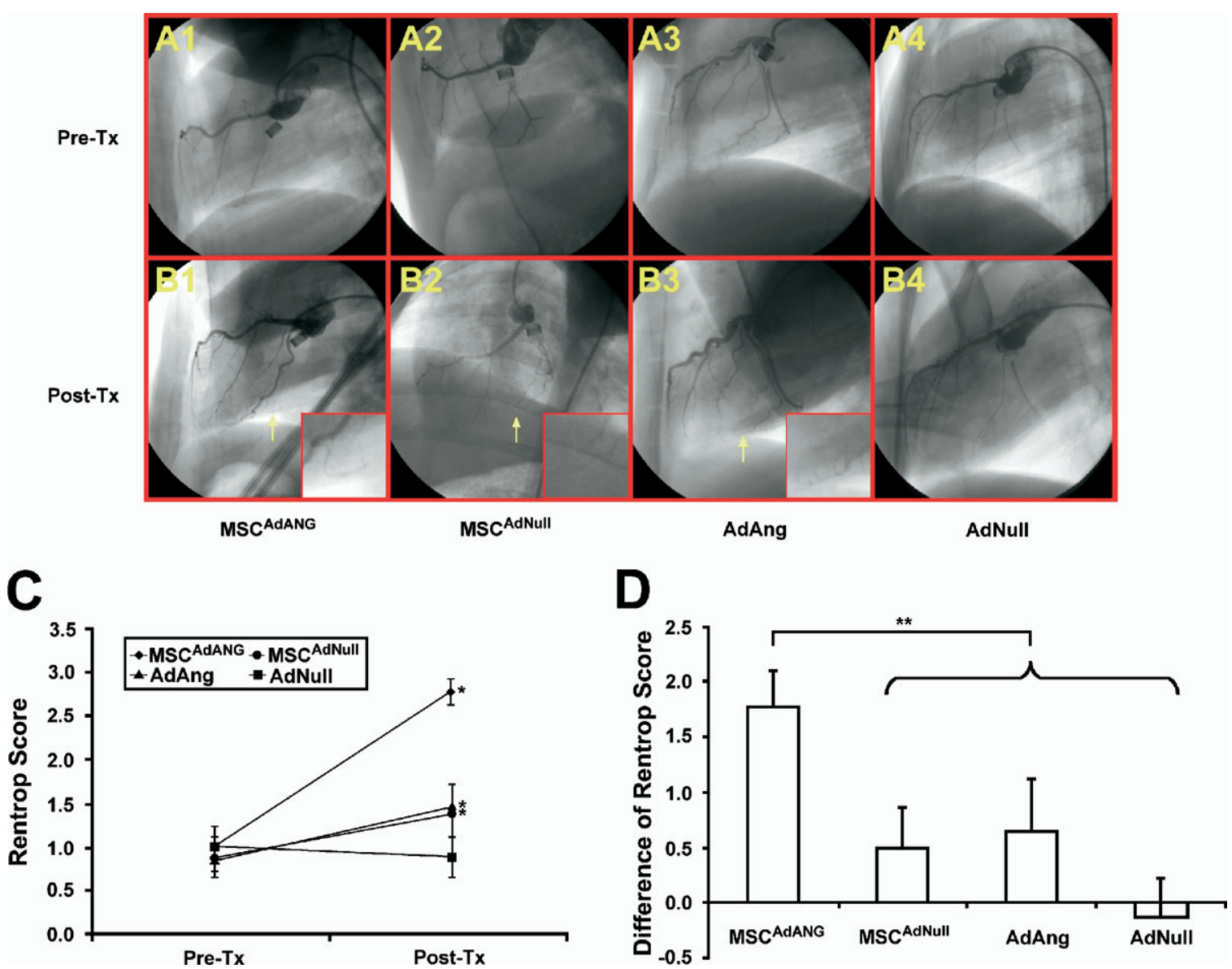

Figure 1. Angiography. A and B, Representative left coronary angiographs conducted 4 weeks after ameroid implantation (Pre-Tx) and 4 weeks after cell or gene implantation (Post-Tx) in pigs implanted with mesenchymal stem cells (MSCs) infected with adenovirus containing the angiogenin gene (MSC ${ }^{A d A n g}$, A1 and B1), with MSCs infected with null adenovirus (MSC ${ }^{A d N u l l}, A 2$ and B2), with angiogenin alone (AdAng, $A 3$ and $B 3$ ), or with null adenovirus alone ( $A d N u l l, A 4$ and $B 4)$. Before transplantation, ischemic areas were observed at the left ventricular wall in all groups. After transplantation, myocardial perfusion (arrows in B1-B4) was greatly enhanced in the MSC $^{\text {AdAng }}$ group (B1), partially enhanced in the MSC $^{\text {AdNull }}$ (B2) and AdAng (B3) groups, and not enhanced in the AdNull group (B4) compared with pretransplantation measures. C, Left coronary angiographic scores (Rentrop scores) were similar among the 4 groups before transplantation. After transplantation, these scores became greatly enhanced $(P<.05)$ in the MSC ${ }^{\text {AdAng }}$ group and partially enhanced $(P<.05)$ in the MSC ${ }^{\text {AdNull }}$ and AdAng groups and remained unchanged in the AdNull group. D, The posttransplantation increase in Rentrop scores was significantly greater in the MSC ${ }^{\text {AdAng }}$ group $(P<.05)$ than in the MSC ${ }^{\text {AdNull }}$ and AdAng groups, whereas the AdNull group displayed a decrease in this measure after treatment. ${ }^{*} \boldsymbol{P}<.05$.

groups at this time point $(P=.783)$. However, 4 weeks after intramyocardial cell or gene implantation (Figure 1, B1-B4), Rentrop scores were significantly greater $(P=.03$ and $P=.02$, respectively) in the MSC $^{\text {AdAng }}$ group than in the MSC $^{\text {AdNull }}$ or AdAng groups, both of which displayed higher scores $(P=.001)$ than the AdNull group (Figure 1, $C$ and $D$ ).

Cardiac function was evaluated immediately before and 4 weeks after cell or gene implantation. Before treatment, LVEF values were similar among the 4 groups. By 4 weeks after treatment, LVEF values had decreased in the AdNull group but improved significantly in the genetically modified cell-treated group (MSC ${ }^{\text {AdAng }}$ ) compared with all other groups $(P<.05)$. LVEF values did not differ significantly between the MSC ${ }^{\text {AdNull }}$ and AdAng groups (Figure 2, A and $B$ ).

Echocardiographic assessment of FAC at the midventricular level did not differ between the MSC ${ }^{\text {AdAng }}$ group and the other groups before treatment. By 4 weeks after treatment, FAC increased significantly in the $\mathrm{MSC}^{\text {AdAng }}$, 
A

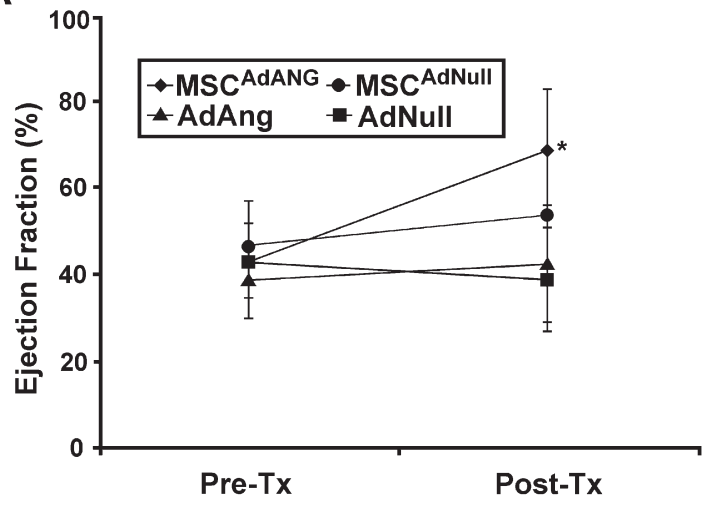

C

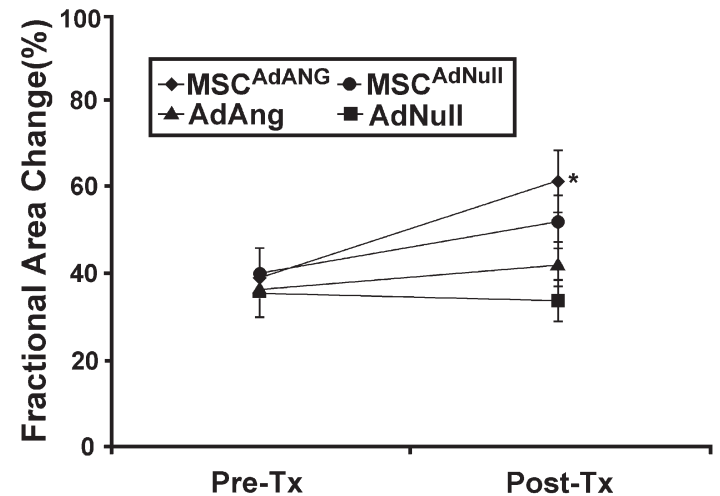

E

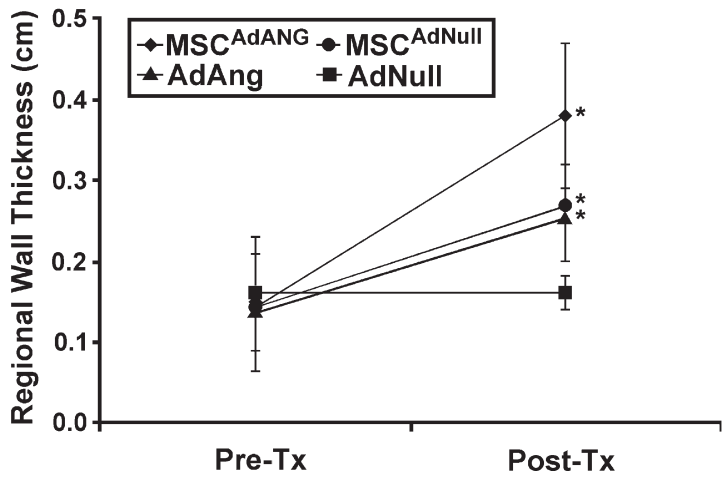

B

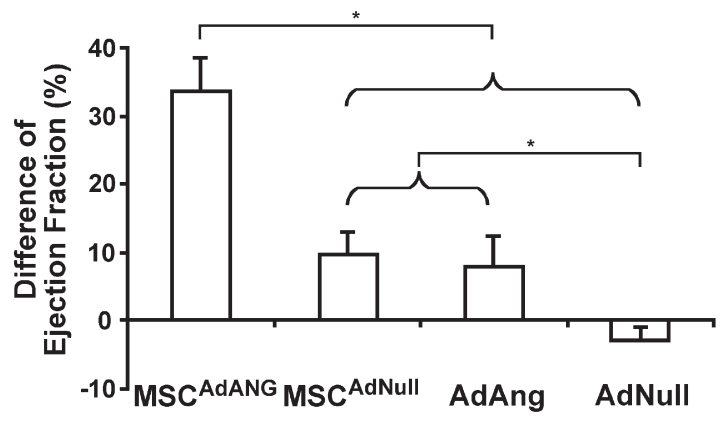

D

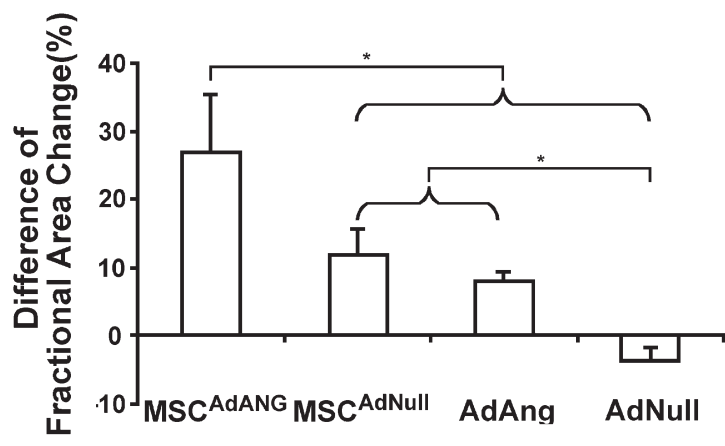

F

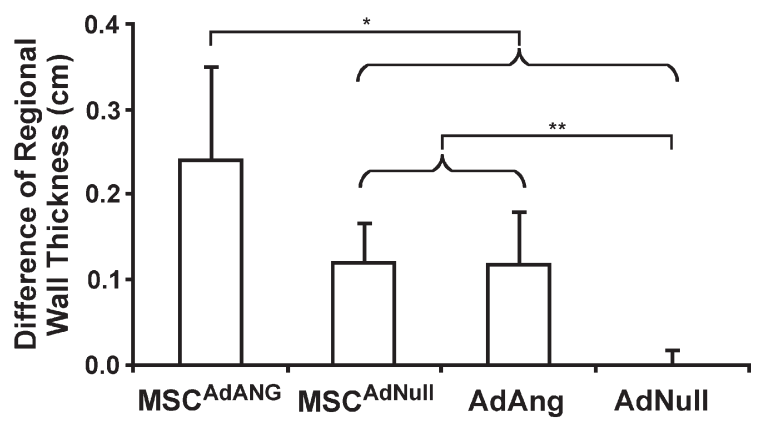

Figure 2. Heart structure and function. A, Left ventricular ejection fraction evaluated by means of echocardiography at 4 weeks after ameroid implantation (Pre-TX) was similar among the 4 groups. Four weeks after cell or gene implantation (Post-Tx), ejection fraction had increased significantly $(P<.05)$ in the group implanted with angiogenin gene-infected mesenchymal stem cells (MSCs; MSC ${ }^{\text {AdAng }}$ group), remained unchanged in the groups implanted with null adenovirus-infected MSCs (MSC ${ }^{\text {AdNull }}$ group) or angiogenin (AdAng group), and decreased in the group implanted with null adenovirus (AdNull group). B, The decrease in ejection fraction from before transplantation to after transplantation was significantly greater $(P<.05)$ in the MSC ${ }^{\text {AdAng }}$ group than in the MSC $^{\text {AdNull }}$ and AdAng groups, both of which showed greater change $(P<.05)$ than in the AdNull group. C, Left ventricular fractional area change measured before transplantation was similar among the 4 groups. After transplantation, fractional area change significantly increased in the MSC ${ }^{\text {AdAng }}$, MSC ${ }^{\text {AdNull, }}$, and AdAng groups but was significantly greater in the MSC ${ }^{\text {AdAng }}$ group compared with that in the control groups $(P<.05)$. D, The decrease in fractional area change from before transplantation to after transplantation was significantly greater in the MSC ${ }^{\text {AdAng }}$ group than in the MSC ${ }^{\text {AdNull }}$ and AdAng groups, both of which showed greater change $(P, .05)$ than the AdNull group. E, Left ventricular wall thickness measured before transplantation was similar among the 4 groups. After transplantation, wall thickness had increased significantly ( $P<.05$ for all groups) in the MSC ${ }^{\text {AdAng }}$,

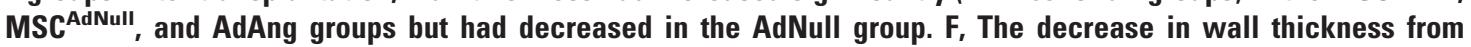
before transplantation to after transplantation was greatest in the MSC ${ }^{\text {AdAng }}$ group $(P<.05$ compared with all other groups) and similar in the MSC ${ }^{\text {AdNull }}$ and AdAng groups $\left(P<.01\right.$ compared with the AdNull group). ${ }^{*} P<.05$, ${ }^{* *} \boldsymbol{P}<.01$. 

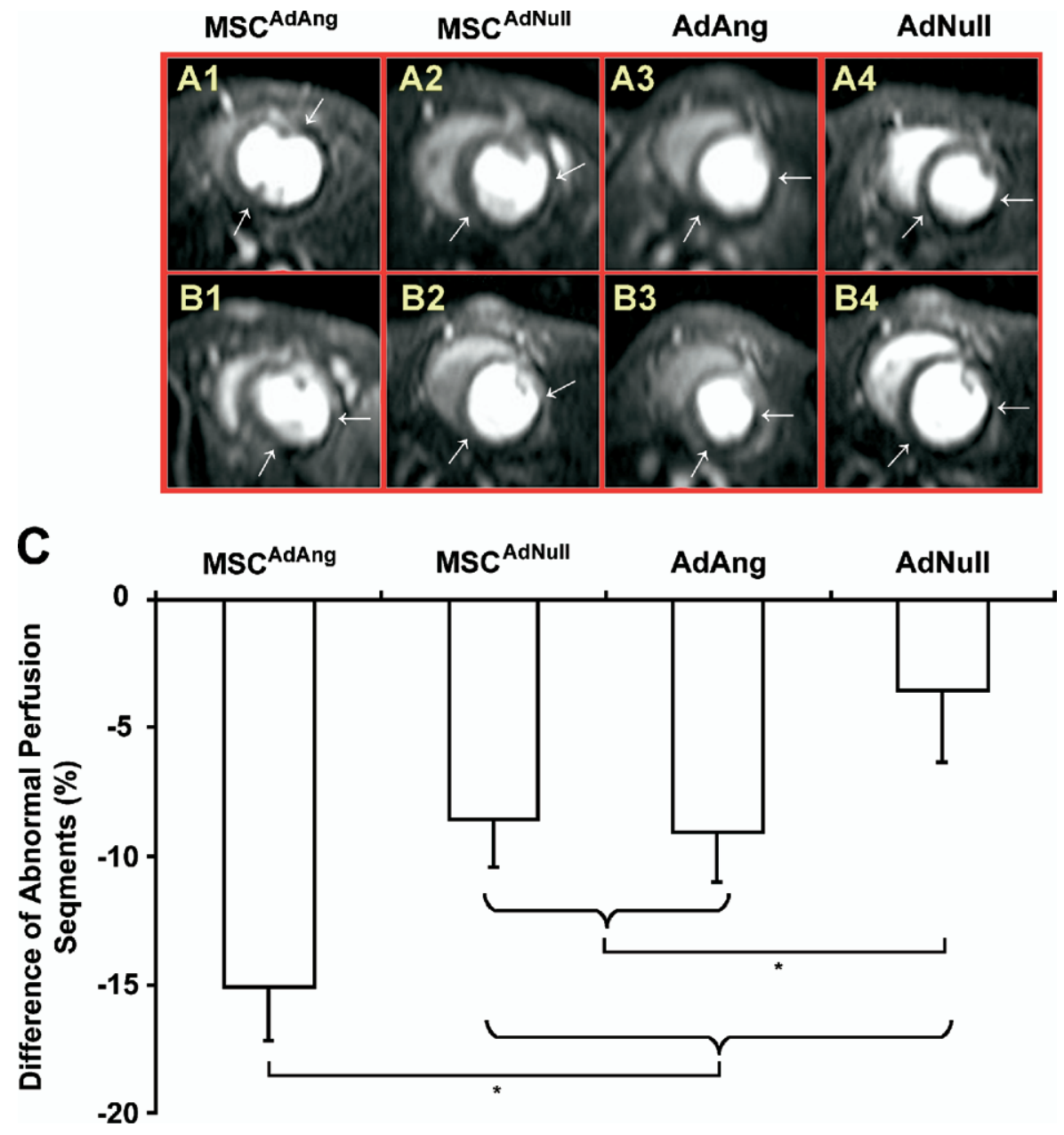

Figure 3. Myocardial perfusion. A and $B$, Magnetic resonance imaging showing myocardial perfusion at 4 weeks after ameroid implantation (before transplantation, A1-A4) and 4 weeks after cell or gene treatment (after transplantation, B1-B4) in pigs implanted with angiogenin gene-infected mesenchymal stem cells (MSCs; MSC ${ }^{\text {AdAng }}$ group), with null adenovirus-infected MSCs (MSC ${ }^{\text {AdNull group), with angiogenin }}$ (AdAng group), or with null adenovirus (AdNull group). There were significant improvements after treatment in the MSC ${ }^{\text {AdAng }}$ (B1), MSC ${ }^{\text {AdNull }}$ (B2), and AdAng (B3) groups and no improvement in the AdNull group (B4). C, From before transplantation to after transplantation, the change in the number of abnormal perfusion segments was greatest in the MSC AdAng group $(P<$ .05 compared with all other groups). Decreases in this measure were similar in the MSC ${ }^{\text {AdNull }}$ and AdAng groups and smallest in the AdNull group $(P<$ .05 compared with the MSC ${ }^{\text {AdNull }}$ and AdAng groups). ${ }^{*} P<.05$.
MSC $^{\text {AdNull, }}$, and AdAng groups $(P<.05)$ but was significantly greater in the MSC AdAng group than in the control groups at this time point (Figure 2, $C$ and $D$ ).

We found that genetically modified cell transplantation (MSC ${ }^{\text {AdAng }}$ group) significantly increased regional wall thickness at 4 weeks after cell or gene implantation $(P<.05$ compared with all other groups). Relative to the AdNull group, wall thicknesses in the MSC $^{\text {AdNull and AdAng }}$ groups also increased significantly after cell or gene implantation $(P<.01$; Figure $2, E$ and $F)$.

Myocardial regional perfusion was evaluated with MRI. The percentages of segments with abnormal perfusion immediately before and 4 weeks after cell or gene implantation, respectively, were as follows: $32.3 \% \pm 4.1 \%$ and $17.2 \% \pm 2.1 \%$ in the MSC AdAng group, $29.7 \% \pm 3.1 \%$ and $21.1 \% \pm 2.0 \%$ in the MSC ${ }^{\text {AdNull }}$ group, $27.7 \% \pm 2.9 \%$ and $18.6 \% \pm 1.9 \%$ in the AdAng group, and $32.1 \% \pm$ $3.4 \%$ and $28.6 \% \pm 2.9 \%$ in the AdNull group (Figure 3, $A$ and $B$ ). Animals that received genetically modified cells (MSC ${ }^{\text {AdAng }}$ group) showed the greatest improvement in regional perfusion after treatment $(P<.05$ compared with all other groups). No significant difference was detected between the cell $\left(\mathrm{MSC}^{\text {AdNull }}\right.$ ) and gene (AdAng) groups, both of which exhibited significantly improved perfusion ( $P<.05$ for both groups) relative to the control (AdNull) group (Figure 3,C).

At the end of the study, hearts were harvested for morphologic study. Lateral transmural infarctions were observed in all hearts in every group (Figure 4, $A-D$ ). Histologic examination of the implanted myocardial region revealed angiogenesis in all groups at 4 weeks after cell or gene treatment (Figure 4, E-H). Infarct sizes were smallest in the MSC $^{\text {AdAng }}$ group $(P<.05$ compared with all other groups $)$, with no significant difference between the $\mathrm{MSC}^{\text {AdNull }}$ and AdAng groups, both of which displayed smaller infarct scars ( $P=.04$ and $P=.03$, respectively) than the AdNull group (Figure 4,I). Vascular density was increased significantly more in animals implanted with genetically modified cells (MSC ${ }^{\text {AdAng }}$ group) than in those given cell (MSC $^{\text {AdNull }}$ group, $P=.04$ ) or gene therapy (AdAng group, $P=.03$ ) 

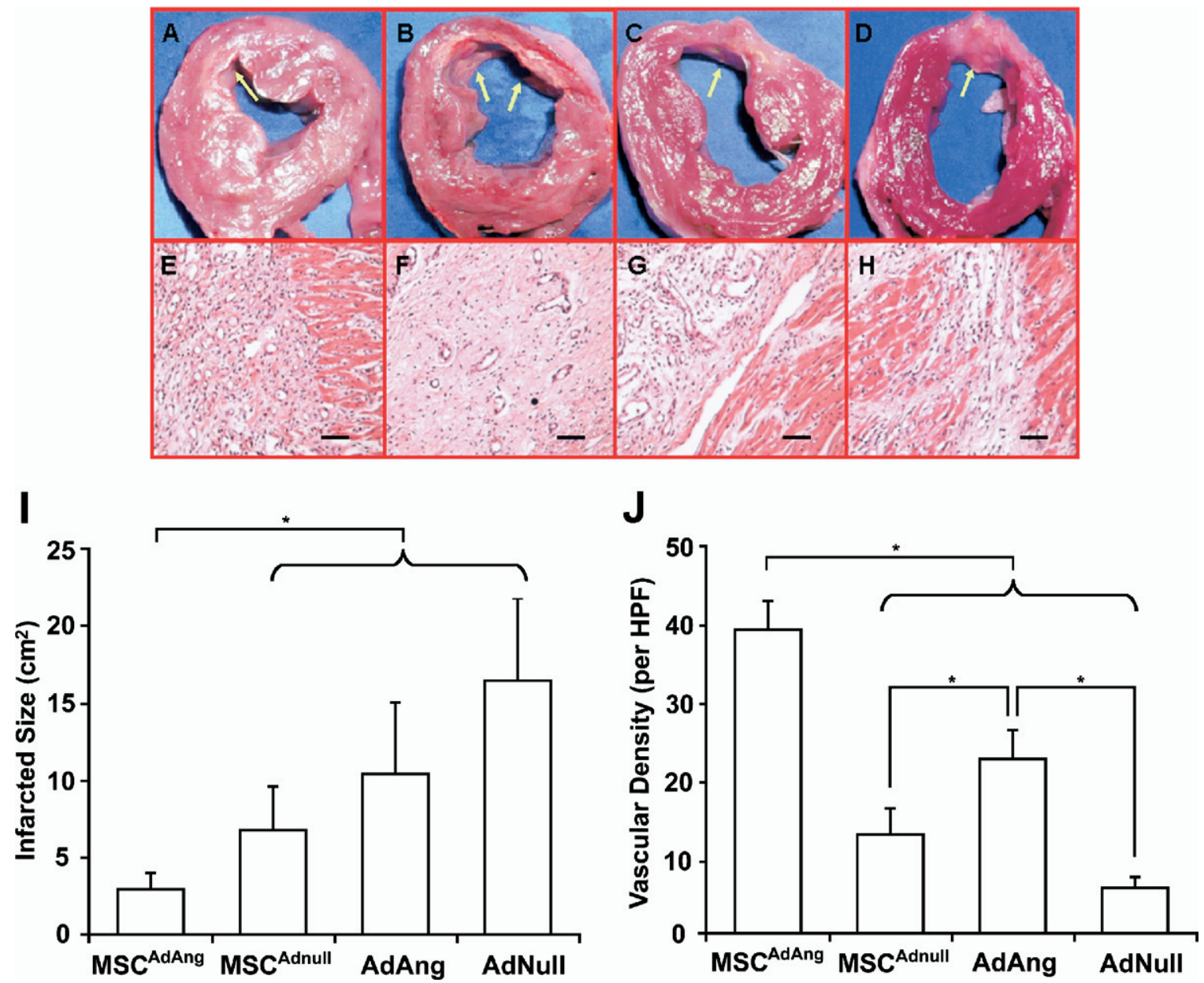

Figure 4. Morphology and histology. A through D, Photographs of left ventricular cross-sections taken at 4 weeks after cell or gene implantation in pigs implanted with angiogenin gene-infected mesenchymal stem cells (MSCs; MSC $^{\text {AdAng }}$ group, A), with null adenovirus-infected MSCs (MSC ${ }^{\text {AdNull }}, \mathrm{B}$ ), with angiogenin gene (AdAng, C), or with null adenovirus (AdNull, D). Lateral infarctions (arrows in A-D) were observed in all groups. $E$ through $H$, Microphotographs illustrating hematoxylin and eosin staining in the infarcted myocardial tissue of each group at 4 weeks after cell or gene implantation. Scale bars $(E-H)=200 \mu \mathrm{m}$. I, the scar area was smallest in the MSC ${ }^{\text {AdAng }}$ group ( $P<.05$ compared with all other groups) and largest in the AdNull group. J, Although vascular density was greatest in the MSC ${ }^{\text {AdAng }}$ group $(P<.05$ compared with all other groups), the AdAng group exhibited more $(P<$ .05) blood vessels than the MSC ${ }^{\text {AdNull }}$ and AdNull groups. ${ }^{*} P<.05$.

alone but was also increased in the AdAng group relative to the $\operatorname{MSC}^{\text {AdNull }}(P=.05)$ and AdNull $(P=.03)$ groups (Figure 4, $J$ ).

Cultured MSCs were labeled with DiI before transplantation to confirm MSC engraftment to the implanted (ischemic) area. Labeling efficiency was $100 \%$ (Figure 5, A). Four weeks after cell implantation, successful MSC engraftment was confirmed in both MSC-treated groups (MSC ${ }^{\text {AdAng }}$ and MSC $^{\text {AdNull }}$ group) by the appearance of DiI-labeled cells at the site of implantation under fluorescence and confocal microscopy (Figure 5, $B-E$ ). A large number of DiI-labeled MSCs were observed in the genetically modified cell- treated group (MSC ${ }^{\text {AdAng }}$ group). These implanted cells were observed in regions of necrotic myocardial tissue and adhered to the collagen-rich matrix, although not in areas remote to the injection site. Immunofluorescent staining for Factor VIII revealed that a significant portion of the implanted MSCs (DiI positive) expressed Factor VIII and were incorporated into blood vessels in the ischemic myocardial regions (Figure 5, $F$ and $G$ ).

Angiogenin expression in the implanted area was quantified by means of Western blot analysis. Animals treated with angiogenin (either through gene or genetically modified cell therapy) displayed significantly greater angiogenin 


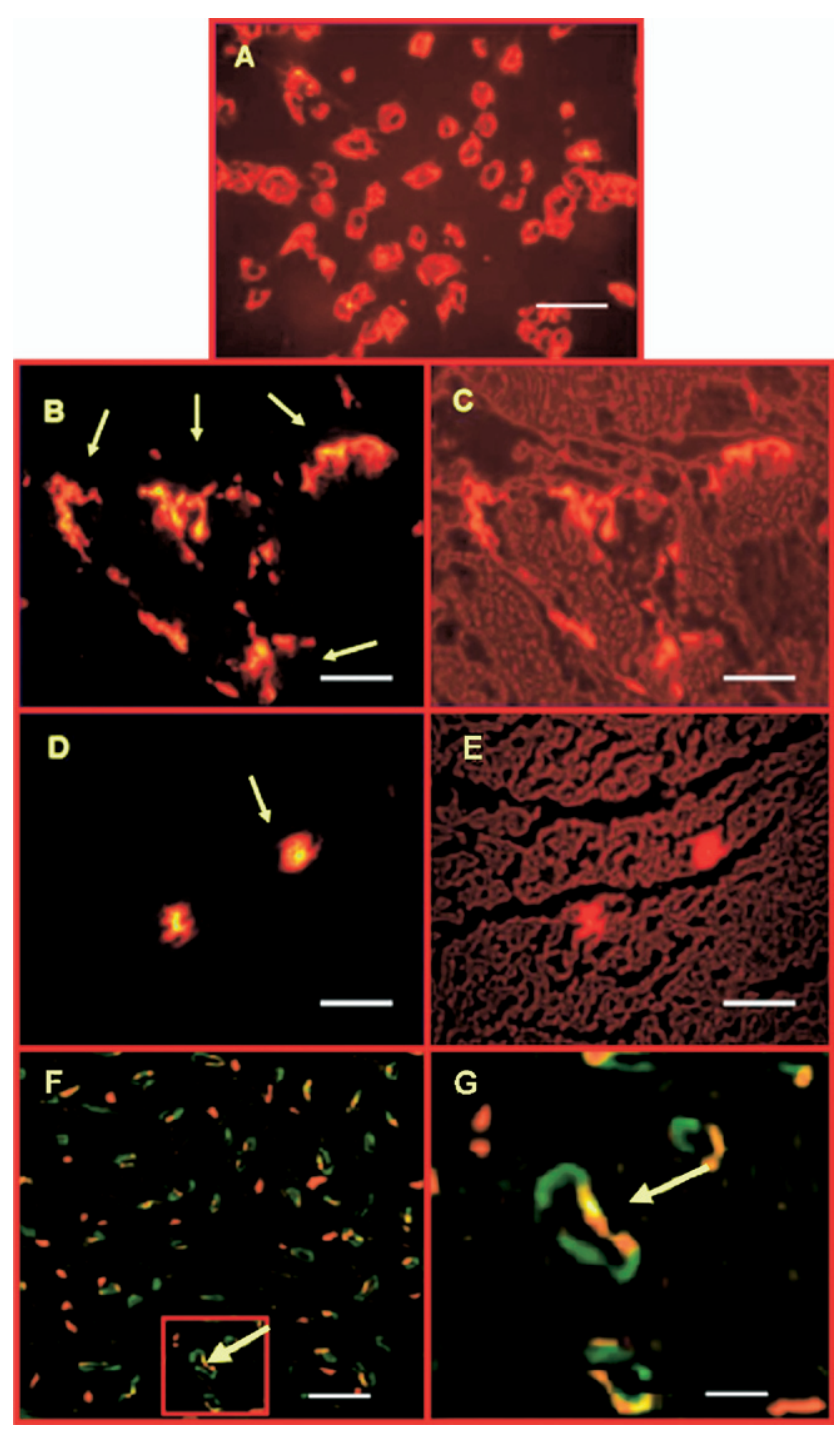

Figure 5. Dil and Factor VIII labeling in the myocardial transplantation area. A, Twenty-four hours after labeling with Dil, $100 \%$ of cultured mesenchymal stem cells (MSCs) were Dil positive under the fluorescent microscope. B through E, Fluorescent and confocal micrographs showing myocardial transplant sites at 4 weeks after cell implantation in pigs implanted with angiogenin geneinfected MSCs (MSC ${ }^{\text {AdAng }}$ group, B and C) or with null adenovirusinfected MSCs (MSC ${ }^{\text {AdNull }}$ group, D and E). The presence of Dil-positive cells (arrows in B and D) indicated successful engraftment of MSCs in both groups. F and G, Confocal micrographs showing Factor VIII staining at myocardial transplant sites in the MSC $^{\text {AdAng }}$ group. Some of the implanted cells were positive for Factor VIII (arrow in F) and were incorporated into blood vessels (arrow in G) in the ischemic regions. Scale bars: $100 \mu \mathrm{m}$ in A and G, $200 \mu \mathrm{m}$ in B through $\mathrm{E}$, and $\mathbf{4 0 0} \mu \mathrm{m}$ in $\mathrm{F}$. protein expression in the implanted area than did non-genetreated groups $(P<.05$ for both groups, Figure 6$)$.

\section{Discussion}

Preclinical $^{19,20}$ and clinical ${ }^{21}$ studies have demonstrated that cell transplantation can augment cardiac function when implanted into the ischemic myocardium. Although the mechanisms responsible for this beneficial effect have not been fully elucidated, the cells increase regional perfusion, maintain myocardial thickness, and prevent ventricular dilatation, which can preserve function and delay heart failure.

In the present study we used ameroid occlusion to produce a pig model of chronic ischemia similar to the status of patients presenting for coronary bypass surgery. The ameroid constrictor results in reproducible and gradual occlusion of the coronary artery with reduced blood flow to the lateral circumflex territory. This reduction in coronary flow leads to a significant reduction in regional myocardial function with minimal myocardial necrosis.

We hypothesized that the synergistic effect of cell and gene therapy would restore cardiac function in the ischemic cardiomyopathic heart. We found that (1) implantation of autologous MSCs overexpressing angiogenin into an ischemic myocardium model significantly improved LV function compared with angiogenin gene therapy or the implantation of MSCs alone; (2) the autologous MSCs engrafted successfully for at least 4 weeks after implantation into the ischemic myocardium, altered pathologic ventricular remodeling, and attenuated contractile dysfunction; and (3) injection of the angiogenin gene in a viral vector into the ischemic myocardium resulted in high expression of angiogenin in the myocardium at 4 weeks after gene injection, which stimulated excellent collateral vessel development. However, this treatment was less effective at improving heart function than the delivery of the gene from the transfected cells.

MSCs were used in the current study as the cell source for combined cell and gene therapy for several reasons. First, MSCs possess pluripotential capabilities ${ }^{6}$ and have been shown to improve heart function in animal studies. ${ }^{8-10}$ Furthermore, these cells are easily obtained from bone marrow and can be expanded in culture. ${ }^{11}$ MSCs have been used as gene carriers in in vivo studies. For example, Duan and associates ${ }^{22}$ reported the transplantation of MSCs transfected with hepatocyte growth factor gene into damaged myocardial tissue in a rat model. In that study the growth factor gene was overexpressed at the implanted area, and cell and gene therapy improved cardiac function.

For this study, we selected angiogenin for transfection into MSCs destined for transplantation. Angiogenin is a basic heparin-binding protein $(14.4 \mathrm{kd})^{14}$ belonging to the RNase superfamily, which shows $33 \%$ homology to ribonuclease $\mathrm{A}^{23}$ It is an angiogenic factor the expression of 
A

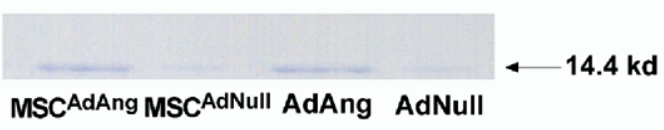

B

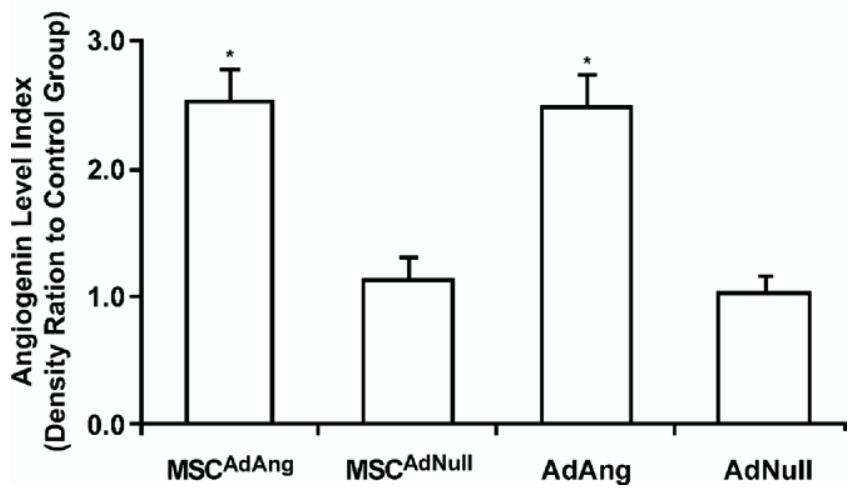

Figure 6. Angiogenin expression. A, Western blot analysis revealed angiogenin protein bands in the myocardial tissues of pigs implanted with angiogenin gene-infected mesenchymal stem cells (MSCs, MSC AdAng group), with null adenovirus-infected MSCs (MSC ${ }^{\text {AdNull }}$ group), with angiogenin gene (AdAng group), or with null adenovirus (AdNull group). B, Quantitative analysis of myocardial angiogenin protein expression, expressed as an index of the ratio of expression relative to AdNull group levels (100\% expression in the AdNull group), indicated that angiogenin protein was expressed at greater levels in the groups that received angiogenin gene transfection (MSC ${ }^{\text {AdAng }}$ and AdAng groups, $P<.05$ for all groups) than in the nontransfected groups (MSC ${ }^{\text {AdNull }}$ and AdNull groups). ${ }^{*} P<.05$.

which is upregulated by hypoxia, ${ }^{24}$ giving it great potential for therapeutic angiogenesis. ${ }^{25}$ Human angiogenin has been localized to chromosome $14 \mathrm{q} 11$, proximal to the $\alpha / \delta$ T-cell receptor. ${ }^{23}$ Several studies have demonstrated that angiogenin stimulates endothelial cell proliferation and promotes the adhesion of endothelial cells and fibroblasts, ${ }^{26,27}$ enhancing neovascular formation. In vivo studies have further shown that angiogenin stimulates collateral vessel development in the ischemic myocardium of rabbit hearts. ${ }^{16}$ In the present study we found that an adenoviral vector containing the angiogenin gene successfully infected the ischemic myocardium after intramyocardial injection. Overexpressed in the myocardium at the injected area, angiogenin stimulated blood vessel formation, which increased regional perfusion and prevented the progress of heart failure in angiogenintreated animals (AdAng group) compared with control animals (AdNull group).

In the animals receiving genetically modified cell transplants (MSC ${ }^{\text {AdAng }}$ group), we demonstrated that a synergistic effect of implanted cells and gene expression contributed to the augmentation of cardiac function. To increase trans- fection efficiency in the MSCs and gene expression in vivo for these animals, we used an adenoviral vector containing angiogenin to transfect the cells. The transfection efficiency was $100 \%$, and Western blot data illustrated that angiogenin was overexpressed in the ischemic myocardium for at least 4 weeks. However, the mechanism responsible for the beneficial effect in combined angiogenin and MSC transplantation and the relative benefits of angiogenin compared with other genes used to enhance cell transplantation (eg, insulinlike growth factor, hepatocyte growth factor, vascular endothelial growth factor, and hypoxia inducible factor 1 alpha) were not identified in this study. Future work will be required to determine the best enhancements to cell transplantation.

Using DiI as a marker, we observed engraftment of transplanted MSCs in the myocardium after cell implantation, and we suspect that the survival of engrafted cells was greater in the group receiving genetically modified MSCs than in the group receiving unmodified MSCs. However, we did not perform a quantitative assessment of cell engraftment, and future studies will need to quantitatively compare our strategy with other gene enhancements to determine the best approach to augment cell engraftment after transplantation.

MRI and coronary angiography detected significant collateral vessel development at the lateral circumflex area after injection of genetically modified MSCs that overexpressed angiogenin. Echocardiographic and MRI results further suggested that the presence of these cells significantly improved functional measures. Alone, MSC implants can induce angiogenesis in vivo because of their ability to secrete various angiogenic factors or cytokines. ${ }^{10,19}$ However, in this study the group receiving MSCs that overexpressed angiogenin demonstrated significantly greater angiogenic capacity than the group undergoing transplantation with untreated cells. The beneficial effect could have been due to angiogenesis, enhanced cell engraftment, or an inhibition of the postinfarction remodeling.

Future studies will be required to determine the duration of transfected gene expression in vivo, although gene expression has been documented to persist in transplanted cells from 5 weeks to 1 year. ${ }^{20}$ Fortunately, even transient gene expression might have lasting effects on angiogenesis, cell engraftment, and the prevention of ventricular remodeling. Future studies will also determine the optimal number of cells for implantation and the optimal dose of angiogenin adenovirus to compare the effectiveness of combination therapy with that of cell or gene therapy under optimal conditions.

In summary, the present study demonstrated that bone marrow-derived MSC transplantation into the ischemic myocardium improved cardiac function by altering ventricular remodeling and increased myocardial perfusion. How- 
ever, transplantation of MSCs overexpressing angiogenin clearly produced stronger beneficial effects on ventricular modulation and the greatest increase in myocardial reperfusion; the combined cell and gene treatment therefore improved cardiac function in a synergistic manner. We suggest that treatments combining cell therapy with gene therapy could be an effective strategy to restore cardiac function in chronic ischemia.

\section{References}

1. Zhang H, Fazel S, Tian H, Mickle DA, Weisel RD, Fujii T, et al. Increasing donor age adversely impacts beneficial effects of bone marrow but not smooth muscle myocardial cell therapy. Am J Physiol Heart Circ Physiol. 2005;289:2089-96.

2. Waksman R, Baffour R. Bone marrow and bone marrow derived mononuclear stem cells therapy for the chronically ischemic myocardium. Cardiovasc Radiat Med. 2003;4:164-8.

3. Kim BO, Tian H, Prasongsukarn K, Wu J, Angoulvant D, Wnendt S, et al. Cell transplantation improves ventricular function after a myocardial infarction: a preclinical study of human unrestricted somatic stem cells in a porcine model. Circulation. 2005;112(suppl):96-104.

4. Elmadbouh I, Chen Y, Louedec L, Silberman S, Pouzet B, Meilhac O, et al. Mesothelial cell transplantation in the infarct scar induces neovascularization and improves heart function. Cardiovasc Res. 2005; 68:307-17.

5. Khurana R, Simons M, Martin JF, Zachary IC. Role of angiogenesis in cardiovascular disease: a critical appraisal. Circulation. 2005;112: 1813-24.

6. Pittenger MF, Mackay AM, Beck SC, Jaiswal RK, Douglas R, Mosca $\mathrm{JD}$, et al. Multilineage potential of adult human mesenchymal stem cells. Science. 1999;284:143-7.

7. Deans RJ, Moseley AB. Mesenchymal stem cells: biology and potential clinical uses. Exp Hematol. 2000;28:875-84.

8. Wang JS, Shum-Tim D, Galipeau J, Chedrawy E, Eliopoulos N, Chiu RC. Marrow stromal cells for cellular cardiomyoplasty: feasibility and potential clinical advantages. J Thorac Cardiovasc Surg. 2000;120: 999-1005.

9. Nishida M, Li TS, Hirata K, Yano M, Matsuzaki M, Hamano K. Improvement of cardiac function by bone marrow cell implantation in rat hypoperfusion heart model. Ann Thorac Surg. 2003;75:768-74.

10. Nagaya N, Kangawa K, Itoh T, Iwase T, Murakami S, Miyahara Y, et al. Transplantation of mesenchymal stem cells improves cardiac function in a rat model of dilated cardiomyopathy. Circulation. 2005;112: 1128-35.

11. Kemp KC, Hows J, Donaldson C. Bone marrow-derived mesenchymal stem cells. Leuk Lymphoma. 2005;46:1531-44.

12. Dib N, McCarthy P, Campbell A, Yeager M, Pagani FD, Wright S, et al. Feasibility and safety of autologous myoblast transplantation in patients with ischemic cardiomyopathy. Cell Transplant. 2005;14: $11-9$.
13. Yaoita H, Takase S, Maruyama Y, Sato Y, Satokawa H, Hoshi N, et al. Scintigraphic assessment of the effects of bone marrow-derived mononuclear cell transplantation combined with off-pump coronary artery bypass surgery in patients with ischemic heart disease. $J$ Nucl Med. 2005;46:1610-7.

14. Kurachi K, Davie EW, Strydom DJ, Riordan JF, Vallee BL. Sequence of the cDNA and gene for angiogenin, a human angiogenesis factor. Biochemistry. 1985;24:5494-9.

15. Shapiro R, Strydom DJ, Olson KA, Vallee BL. Isolation of angiogenin from normal human plasma. Biochemistry. 1987;26:5141-6.

16. Fett JW, Strydom DJ, Lobb RR, Alderman EM, Bethune JL, Riordan $\mathrm{JF}$, et al. Isolation and characterization of angiogenin, an angiogenic protein from human carcinoma cells. Biochemistry. 1985;24:5480-6.

17. Balci B, Yilmaz O. Extent of coronary collateral vessel decrease with advanced age. Acta Cardiol. 2004;59:431-4.

18. Cerqueira MD, Weissman NJ, Dilsizian V, Jacobs AK, Kaul S, Laskey WK, et al. American Heart Association Writing Group on Myocardial Segmentation and Registration for Cardiac Imaging. Standardized myocardial segmentation and nomenclature for tomographic imaging of the heart. A statement for healthcare professionals from the Cardiac Imaging Committee of the Council on Clinical Cardiology of the American Heart Association. Int $J$ Cardiovasc Imaging. 2002;18:539-42.

19. Li RK, Jia ZQ, Weisel RD, Mickle DA, Zhang J, Mohabeer MK, et al. Cardiomyocyte transplantation improves heart function. Ann Thorac Surg. 1996;62:654-61.

20. Atkins BZ, Hueman MT, Meuchel JM, Cottman MJ, Hutcheson KA, Taylor DA. Myogenic cell transplantation improves in vivo regional performance in infarcted rabbit myocardium. J Heart Lung Transplant. 1999;18:1173-80.

21. Heeschen C, Lehmann R, Honold J, Assmus B, Aicher A, Walter DH, et al. Profoundly reduced neovascularization capacity of bone marrow mononuclear cells derived from patients with chronic ischemic heart disease. Circulation. 2004;109:1615-22.

22. Duan HF, Wu CT, Wu DL, Lu Y, Liu HJ, Ha XQ, et al. Treatment of myocardial ischemia with bone marrow-derived mesenchymal stem cells overexpressing hepatocyte growth factor. Mol Ther. 2003; 8:467-74.

23. Adams SA, Subramanian V. The angiogenins: an emerging family of ribonuclease related proteins with diverse cellular functions. Angiogenesis. 1999;3:189-99.

24. Hartmann A, Kunz M, Kostlin S, Gillitzer R, Toksoy A, Brocker EB, et al. Hypoxia-induced up-regulation of angiogenin in human malignant melanoma. Cancer Res. 1999;59:1578-83.

25. Greenway MJ, Alexander MD, Ennis S, Traynor BJ, Corr B, Frost E, et al. A novel candidate region for ALS on chromosome 14q11.2. Neurology. 2004;63:1936-8.

26. Hu G, Xu C, Riordan JF. Human angiogenin is rapidly translocated to the nucleus of human umbilical vein endothelial cells and binds to DNA. J Cell Biochem. 2000;76:452-62.

27. Distler JH, Hirth A, Kurowska-Stolarska M, Gay RE, Gay S, Distler O. Angiogenic and angiostatic factors in the molecular control of angiogenesis. $Q J$ Nucl Med. 2003;47:149-61. 